\title{
DIAGNÓSTICO DE FALTAS EM SISTEMAS DE POTÊNCIA: DEFINIÇÃO DO PROBLEMA E ABORDAGENS VIA INTELIGÊNCIA ARTIFICIAL
}

\author{
Ghendy Cardoso $\mathbf{J r}^{\dagger}$ \\ ghendy@ufpa.br
}

Jacqueline G Rolim*

\author{
Hans Helmut Zürn*
}

*Grupo de Sistemas de Potência -Departamento de Engenharia Elétrica, Universidade Federal de Santa Catarina, 88040-900, Florianópolis, SC

${ }^{\dagger}$ NESC - Núcleo de Energia, Sistemas e Comunicações, Universidade Federal do Pará

\begin{abstract}
Due to the continuous increase in energy demand, without the corresponding investment in generation and transmission capacity, power systems have been operating close to their limits. Thus, the use of tools for supporting the operation is essential in order to increase reliability, reducing the number and/or duration of non-programmable interruptions, optimizing the use of assets. Aiming to reduce the duration of interruptions, several research works have been published describing tools developed to support the fault diagnosis task. These tools would be used during real time operation, after contingencies with definitive interruptions before starting the restoration process. This paper discusses important aspects of the fault diagnosis problem and the techniques more frequently employed to model and tackle this problem.
\end{abstract}

KEYWORDS: Fault Diagnosis, Power Transmission Systems, and Artificial Intelligence.

\section{RESUMO}

O constante aumento na demanda, sem o correspon-

\footnotetext{
Artigo submetido em $14 / 08 / 02$

1a. Revisão em 28/04/03

Aceito sob recomendação do Ed. Assoc. Prof. Glauco Taranto
}

dente aumento na capacidade de geração e transmissão de energia elétrica, tem levado os sistemas de potência a operarem cada vez mais próximos dos seus limites. Este fato torna imprescindível o uso de ferramentas de apoio à operação, capazes de aumentar a confiabilidade do sistema, reduzindo o número e/ou duração de paradas não programadas das instalações. Na tentativa de reduzir a duração das interrupções, têm sido publicados trabalhos envolvendo ferramentas desenvolvidas para uso na operação em tempo real, com a finalidade de dar apoio ao diagnóstico de faltas. Tais ferramentas seriam empregadas após contingências com desligamentos definitivos, antes de dar início ao processo de recomposição da rede. Este artigo discute aspectos importantes do diagnóstico de faltas e as técnicas mais utilizadas para modelar e tratar este problema.

PALAVRAS-CHAVE: Diagnóstico de faltas, Sistemas de Transmissão, Inteligência Artificial.

\section{INTRODUÇÃO}

Em sistemas elétricos de potência um grande número de mensagens e alarmes é transmitido ao centro de controle após a ocorrência de distúrbios. Tais distúrbios são provocados por diferentes tipos de faltas, podendo ocorrer em qualquer parte do sistema. 
Os equipamentos de proteção são responsáveis por detectar a ocorrência de um defeito e agir apropriadamente de modo a isolar somente a parte defeituosa do sistema (seletividade). Para que o restabelecimento do sistema ocorra o mais rapidamente possível, de modo a evitar danos aos consumidores e à empresa fornecedora, é essencial que a estimação dos eventos que produziram uma determinada seqüência de alarmes ocorra de forma rápida, precisa e segura (Coutto Filho et al., 1999).

Atuações incorretas da proteção, assim como problemas de oscilações e sobretensões após a ocorrência da falta levam a desligamentos em grandes proporções (Gomes et al., 2002), que dificultam a avaliação pelos operadores sobre a causa inicial dos desligamentos. Outros problemas tais como falhas em unidades terminais remotas (UTR), nos canais de comunicação, ou na aquisição de dados, implicam em informação incompleta ou corrompida, o que dificulta ainda mais a tarefa de diagnóstico.

Cabe ressaltar que, além desta análise em tempo real da causa dos desligamentos, uma análise mais detalhada é feita posteriormente (análise pós-morte) por engenheiros de proteção, que trabalham com as informações da seqüência de eventos da contingência e também com curvas de corrente e tensão registradas por oscilógrafos ou registradores digitais de perturbações. Este processo, realizado off-line, é bastante trabalhoso e requer considerável conhecimento e habilidade humana (Chairman et al., 1998).

O raciocínio temporal é considerado por Vale \& Ramos (1995) como chave na solução de problemas em tempo real. Isto, pois questões temporais fazem parte do processo de solução utilizado pelos operadores e pelos engenheiros de proteção, ou seja, as conclusões obtidas pelos especialistas dependem dos tempos de ocorrência e da ordem cronológica dos eventos, além dos intervalos de tempo que separam os diversos eventos.

O processo de automatização da análise de operação de disjuntores e relés tem sido motivo de pesquisa há mais de trinta anos (Dyliacco \& Kraynak, 1969).

O desenvolvimento de uma ferramenta computacional para diagnóstico on-line de faltas em sistemas de potência está longe do trivial, dado o volume de informações e incertezas das informações disponíveis ao operador, além do estresse e a urgência da situação (Rodriguez et al., 1996).

A tarefa de identificar os eventos que produzem uma determinada seqüência de alarmes consiste basicamente de 3 funções: gerenciamento de alarmes, diagnóstico de faltas e análise pós-morte.
O processador de alarmes é uma ferramenta que visa filtrar alarmes redundantes. Sua função é agrupar, selecionar e apresentar ao operador apenas os alarmes mais importantes (Murta Vale et al., 2002)

Por outro lado, o diagnóstico de faltas busca encontrar a causa ou as causas que deram origem a determinados alarmes. É importante que os dados utilizados por esta função sejam, de preferência, gerados pelo módulo de processamento de alarmes. O módulo de diagnóstico de faltas deve apresentar grande sucesso em estimar a seção do sistema (linha de transmissão, barramento ou transformador) que deu origem aos desligamentos, além de identificar a atuação imprópria de relés e disjuntores (Fu et al., 1995). Este módulo exige o uso ferramentas computacionais adequadas à operação em tempo real, uma vez que este é realizado on-line.

A etapa de análise pós-morte corresponde a uma pesquisa bastante detalhada sobre as causas da falta, além de identificar possíveis falhas ou confirmar a correta operação de relés e disjuntores. Por ser uma fase bastante trabalhosa e que exige grande precisão, onde são utilizados todos os tipos de dados disponíveis, esta é normalmente realizada off-line.

Os sistemas especialistas, as redes neurais, a lógica difusa, os algoritmos genéticos, a busca tabu e as redes de Petri constituem as principais técnicas aplicadas ao problema de diagnóstico de faltas.

\section{DEFINIÇÃO DO PROBLEMA}

Durante grandes contingências, a operação de relés de proteção e disjuntores espalhados pelo sistema elétrico dá origem a um grande volume de mensagens de alarmes, que dependendo da disponibilidade do sistema de comunicação são enviados, juntamente com outros valores supervisionados, aos centros de controle do sistema (Handschin et al., 1996).

Após a ocorrência de distúrbios com desligamentos definitivos, a prioridade é restaurar o sistema elétrico atingido. No entanto, antes desta etapa é necessário:

Identificar o(s) componente(s) que estão em falta (selecionar a hipótese mais provável);

- Efetuar manobras para isolar o componente com defeito, caso seja necessário inspeção por parte das equipes de manutenção;

- Restaurar as partes do sistema atingidas pelo desligamento, mas que não estão em falta; 
- Caso haja necessidade, as equipes de manutenção são deslocadas para que o defeito possa ser corrigido (isto geralmente ocorre quando algumas proteções operam, bloqueando o equipamento protegido);

- Finalmente, restaurar a(s) parte(s) onde ocorreu o defeito.

Destas etapas, a primeira corresponde ao diagnóstico de faltas. Com a seleção da hipótese correta, consegue-se minimizar o tempo de interrupção e o risco de agravar a situação ou danificar equipamentos, religando-os indevidamente.

Uma grande variedade de dispositivos de proteção é instalada no sistema elétrico, dependendo do equipamento a ser protegido e do seu nível de tensão. Cada tipo de dispositivo de proteção é dotado de certos critérios capazes de detectar o estado anormal do componente elétrico.

A aplicabilidade de um sistema de diagnóstico de faltas depende de diferentes tipos de relés de proteção a serem considerados (Handschin et al., 1996):

- Relés diferenciais (transformadores, geradores);

- Relés de distância (linhas);

- Relés de sobrecorrente instantâneo ou temporizado, com ou sem unidade direcional (linhas e transformadores);

- Relés de sobrecorrente de neutro temporizado, com ou sem unidade direcional (linhas e transformadores).

Geralmente, os relés instantâneos protegem o equipamento, enquanto que as unidades temporizadas servem como retaguarda.

Outros tipos de relés, tais como: Buchholz e temperatura, no caso de transformadores; relés da teleproteção, no caso das linhas (recepção e partida da teleproteção, chave de bloqueio da portadora, etc.); e relés utilizados em disjuntores (baixa pressão de SF6, discordância de pólos, etc.), também podem ser considerados. O tipo e a quantidade de informações disponíveis dependem exclusivamente do sistema elétrico em consideração.

Problemas inerentes à tarefa de diagnóstico de faltas envolvendo relés, disjuntores, canais de comunicação, entre outros, devem ser levados em consideração. A metodologia de solução a ser implementada deve ser escolhida levando-se em conta os seguintes fatores:
- Falhas em relés ou disjuntores, e faltas múltiplas, complicam o processo de diagnóstico de faltas. No primeiro caso a falta é eliminada através da proteção de retaguarda, implicando em uma grande área desligada. No segundo caso, o número de combinações possível torna o problema de diagnóstico bastante complexo, devido a sua natureza combinatória;

- Aquisição de dados corrompidos;

- Falha no sistema de transmissão de dados e unidades terminais remotas;

- Perda de informações, quando o volume de dados a ser transmitido for muito grande. Esta situação pode surgir durante a ocorrência de falta extremamente grave com muitos desligamentos;

- Nem todas as subestações possuem registro de seqüência de eventos;

- Imprecisão nos tempos de ocorrência dos eventos;

- Alarmes que surgem pela ocorrência de eventos secundários podem ser facilmente misturados com alarmes causados pela perturbação primária;

- Dificuldade em simular eventos raros e falta de dados históricos prejudica o progresso de trabalhos na área, uma vez que esses compõem a base para o desenvolvimento de modelos;

- A experiência do operador só pode ser obtida na prática, mas faltas severas ocorrem poucas vezes. A experiência adquirida sobre uma determinada falta, nem sempre é aplicável sobre a ocorrência de outras (Fu et al., 1995).

Por fim, o diagnóstico de faltas ou estimação da seção em falta é definido como um problema de tomada de decisão, onde várias hipóteses (seções em falta), previamente formuladas, competem entre si, cabendo ao operador ou à ferramenta computacional de apoio, selecionar a mais provável (Park et al., 1999).

\section{SISTEMA DE AQUISIÇÃO DE DADOS}

Os sistemas SCADA (Supervisory Control and Data Acquisition) e/ou EMS (Energy Managment Systems) são as principais fontes de informações disponíveis aos operadores do centro de controle, as quais possibilitam um diagnóstico on-line, antes da etapa de restauração do sistema. Tipicamente, estes sistemas são compostos por computadores para processamento dos dados, unidades terminais remotas (UTR) espalhadas geograficamente 
para coleta e envio dos dados das subestações e usinas, sistemas de comunicação, e interfaces homem-máquina.

As informações disponíveis aos operadores (seqüência de eventos e topologia do sistema) também são empregadas pelos engenheiros de proteção, para produção de um diagnóstico mais preciso off-line. Este diagnóstico também verifica, além da causa da falha, se o comportamento dos dispositivos de proteção foi adequado. Estes engenheiros contam também com informações coletadas pelos oscilógrafos, ou pelos registradores digitais de perturbação (RDP). Este conjunto de dados é essencial para a avaliação da operação da proteção, mas durante condições extremas podem produzir uma sobrecarga de informações (Bell et al., 1998).

Um dos maiores problemas relativos ao registrador digital de perturbações está relacionado ao grande número de perturbações e dados registrados. Tal problema pode ser contornado com o desenvolvimento de rotinas capazes de distinguir faltas transitórias e outros eventos transitórios que não representem uma falta permanente.

No Brasil, as companhias energéticas estão substituindo os oscilógrafos convencionais pelos registradores digitais de perturbações, resultando em benefícios à operação, entre os quais citam-se (Gomes et al., 1999):

- Operação confiável (confiabilidade dos dados referentes a uma perturbação);

- Banco de dados em meios computadorizados (histórico com acesso rápido);

- Gerenciamento integrado com os registros provenientes da proteção digital;

- Centralização das informações;

- Função de localização de defeitos - simulações realizadas pelos engenheiros da ELETROSUL, mostram um erro médio de $3,66 \%$, o que representa um bom desempenho desta função (Terreros et al., 1999).

\section{DIAGNÓSTICO DE FALTAS EM SISTE- MAS DE DISTRIBUIÇÃO: UMA BREVE REVISÃO}

Os sistemas de transmissão, constituídos por linhas de transmissão e subestações de alta e extra-alta tensão, são normalmente gerenciados pelos sistemas SCADA e/ou EMS. A monitoração em tempo real desses sistemas viabiliza a implementação de ferramentas computacionais para diagnosticar faltas. A maioria dos artigos publicados nesta área utiliza informações sobre os estados de relés e disjuntores (Rodrigo et al., 1996).

Por outro lado, os sistemas de distribuição não são tão bem monitorados. Em muitos sistemas de distribuição existentes, não há a disponibilidade de registradores de seqüência de eventos e tomadas de tempos precisas sobre a operação de relés e disjuntores (Teo \& Gooi, 1998). A monitoração destes sistemas geralmente se restringe a relés e disjuntores até a saída de baixa tensão das subestações.

Devido à falta de informações, a tarefa de diagnóstico em sistemas de distribuição operando em $23 \mathrm{kV}$ ou menos é bastante diferente daquela em tensões mais altas. Geralmente, os operadores tomam consciência da ocorrência da falta ao receberem reclamações, via telefone, dos consumidores. Tal fato implica em uma grande demanda de tempo por parte do operador para tomar conhecimento e identificar a causa dos desligamentos em situações de emergência.

Além disso, as perturbações em sistemas de distribuição normalmente ocorrem em condições críticas de tempo e, ao contrário dos sistemas de transmissão, muitas faltas podem ser geradas simultaneamente, sobrecarregando os engenheiros de distribuição, que rapidamente recebem um grande número de mensagens, relatórios de danos e reclamações telefônicas realizadas pelos consumidores (Montakhab \& Adams, 1998).

$\mathrm{Na}$ maioria dos sistemas de distribuição são utilizados métodos manuais para determinar a causa dos desligamentos. É bastante comum o envio de uma equipe ao campo para realizar inspeções visuais nos alimentadores, sendo a comunicação entre o operador e a turma de campo realizada por meio de rádios. A implementação do sistema SCADA e a automação dos sistemas de distribuição possibilitariam o uso de programas computacionais para apoiar os operadores na tarefa de determinar a seção defeituosa que deu origem aos desligamentos (Rodrigo et al., 1996).

Alguns artigos publicados na área de diagnóstico em sistemas de distribuição, devido à falta de dados adicionais, utilizam informações sobre o mapeamento de reclamações realizadas por consumidores via telefone e instalação de sensores ao longo da rede. Chang \& Wen (1998) utilizam estas informações e apresentam um método que leva em consideração a localização, seletividade e as possibilidades de operação do sistema de proteção em sistemas de distribuição de baixa tensão. O dispositivo de proteção mais provável de ter operado é encontrado através de uma eficiente busca, utilizando-se para tal, o método de busca tabu. 
O trabalho desenvolvido por Mohamed \& Rao (1995), além de detectar a falta e estimar a seção em que esta ocorreu, classifica o tipo da falta, incluindo a abertura de condutores (mono ou bipolar). A cada uma das funções é associada uma rede neural treinada pelo algoritmo de retropropagação. Cada conjunto de treinamento é composto pela corrente e tensão de fase e seqüência da subestação à qual está ligado o alimentador a ser monitorado. Durante o treinamento, são considerados vários níveis de carga (20\%-100\%) e diversos eventos (operação normal e anormal - falta de baixa e alta impedância). Com a divisão do alimentador de $24 \mathrm{kV}$ em três seções a serem investigadas, o solo é classificado como, úmido, moderado, ou seco, sendo cada uma dessas classificações subdivididas em três níveis de condutibilidade (baixa, média e alta). Durante o treinamento são utilizados 160, 384 e 531 padrões, de modo a representar a operação normal, anormal de baixa e alta impedância, respectivamente.

Em sistemas de distribuição, falta monofásica, bifásica e trifásica com baixa impedância de falta apresentam correntes suficientes para operar os dispositivos de proteção. Em contrapartida, faltas monofásicas em sistemas conectados em delta (não aterrados), e de alta impedância (com formação de arco) em sistemas em estrela aterrados, nem sempre resultam em corrente suficiente para operar o dispositivo de proteção (Buttler et al., 1997).

Buttler et al. (1997) utilizam informações sobre as correntes de fase (A, B e C) e de neutro (em sistemas aterrados), além do conhecimento do especialista sobre o comportamento dessas variáveis mediante a ocorrência de uma falta. A partir de tal conhecimento, uma rede neural foi treinada para classificar a fase e a provável distância da falta. Quatro tipos diferentes de falta monofásica são considerados: condutor não rompido (condutor em contato com o chão, árvores, etc.), condutor rompido tocando o chão no lado da subestação, condutor rompido tocando o chão no lado da carga, e abertura monopolar do condutor sem nenhum lado em contato com o chão.

Detecção de defeitos em alimentadores de distribuição, com alta impedância de falta, também, foi motivo de pesquisa para Jota \& Jota (1998). A técnica proposta consiste em analisar a resposta do alimentador a impulsos de ondas periodicamente injetadas no início do mesmo. As respostas são comparadas com padrões previamente armazenados em um banco de dados, sendo estes obtidos com o alimentador operando normalmente para as diversas configurações possíveis. O sistema proposto processa a informação adquirida do alimentador com aquela contida na base de dados, utilizando a máquina de inferência difusa para indicar a possível ocorrência de anomalias.

\section{DIFERENÇAS ENTRE DIAGNÓSTICO EM NÍVEL DE SUBESTAÇÃO E DE CENTRO DE OPERAÇÃO}

O diagnóstico em nível de subestação é de algum modo bastante similar àquele realizado em nível de Centro de Operações. Geralmente, a base de conhecimento é relacionada à topologia da rede, configuração da subestação, lógica de operação de relés e regras de natureza heurística. Segundo Hertz \& Fauquembergue (1992) as principais diferenças entre o diagnóstico em nível de subestação e centralizado são:

Em nível de subestação:

- Pode contemplar mais detalhes em razão da disponibilidade de um maior número de dados, já que nem todos são enviados aos centros de controle;

- Disponibilidade de dados analógicos ou digitais sobre correntes e tensões de fase, possibilitando analisar o tipo de falta, calcular a distância da falta, e analisar faltas transitórias de alta freqüência;

- Necessidade de um recurso para obtenção de dados em subestações vizinhas;

- Pode exigir um nível de conhecimento mais aprofundado do que heurístico, uma vez que a funcionalidade dos dispositivos contidos na subestação pode ser formalmente transcrita.

\section{Em nível Centralizado:}

- Necessidade de um módulo para selecionar a parte do sistema a ser analisada, isto é, identificar as partes do sistema atingidas pela falta;

- Necessidade de compactação e grande capacidade de transmissão de dados (em grande volume, se for feita uma análise semelhante à que seria realizada em nível de subestação).

Ambos os tipos de diagnóstico contam ainda com:

- Seqüência de tempo em que os eventos ocorrem, permitindo analisar o desempenho dos dispositivos de proteção, além de ajudar no processo de localização de faltas (neste caso, os relógios das subestações devem estar sincronizados); 
- Cuidados na manutenção da base de dados, pois embora existam muitos dados, estes podem ser inúteis caso não sejam tratados adequadamente.

\section{MÉTOdOS DE SOLUÇÃO}

A literatura propõe a utilização de sistemas inteligentes na tarefa de diagnóstico de faltas. A utilização das técnicas de inteligência artificial (IA) é necessária devido à ausência de uma formulação analítica eficaz, capaz de solucionar o problema.

Entre as diversas técnicas de IA, destacam-se os sistemas especialistas, as redes neurais, a lógica difusa e os algoritmos genéticos. A aplicação de outros métodos, tais como redes de Petri, também é encontrada na literatura.

Tais técnicas surgem como soluções promissoras, onde a natureza heurística e simbólica do raciocínio envolvido nas tarefas do operador pode ser modelada. Os resultados obtidos com aplicações de sistemas inteligentes em sistemas de potência mostram que a utilização de técnicas de inteligência artificial é adequada e bastante proveitosa, capaz de solucionar problemas nos quais as técnicas tradicionais de programação não apresentam bons resultados (Vale \& Ramos, 1995).

Em sistemas elétricos de potência, após a ocorrência de uma falta, o operador necessita selecionar as mensagens mais relevantes, extrair uma conclusão a partir dos dados disponíveis, e agir apropriadamente de modo a restabelecer o sistema ao seu estado seguro. Cabe ao operador decidir se pode ou não religar os equipamentos atingidos pelo desligamento. A disposição de uma ferramenta computacional de auxílio à tomada de decisão, juntamente com o sistema de supervisão, poderá tornar mais rápida esta tarefa e diminuir os riscos causados por uma má interpretação dos eventos sinalizados, principalmente nos casos de sobrecarga de informações.

\subsection{Sistemas especialistas}

Sistemas especialistas (SE's) são programas de computador que armazenam o conhecimento de especialistas em um determinado domínio e o utilizam, combinado a métodos de inferência, para resolver problemas que são suficientemente complexos e/ou de difícil modelagem por meio de métodos analíticos convencionais (Feigenbaum, 1982).

A forma mais comum de armazenar o conhecimento heurístico é a das regras de produção. Neste caso, o conhecimento extraído dos especialistas dá origem a uma base de conhecimentos representada na forma: se (condição)... então (conclusão/ação). Outra alternativa é a dos sistemas baseados em casos, que "trabalham" com casos ocorridos, de forma similar ao que acontece com uma pessoa, que vai adquirindo experiência no decorrer dos anos e que relembra ocorrências passadas para resolver novos problemas (Lefévre, 1996).

Segundo Rodriguez et al. (1996) o sistema especialista é uma das técnicas de solução para o diagnóstico de faltas mais escolhida pelos pesquisadores, desde que Wollenberg (1986) sugeriu a sua utilização no tratamento de alarmes. Embora muito requisitado pelos pesquisadores, poucas pesquisas foram totalmente desenvolvidas e aplicadas em sistemas reais. Entre os trabalhos implementados em campo, Rodriguez et al., destacam o LAIDA, desenvolvido para a empresa de energia espanhola Iberdrola, em operação desde 1989.

O uso de sistemas especialistas no diagnóstico de faltas é praticamente inevitável, uma vez que o operador necessita de resultados diretos na forma simbólica, fáceis de serem analisados (Chiganer et al., 2000). Conseqüentemente, embora sejam utilizados outros métodos de inteligência artificial na solução do problema, faz-se necessário o sistema especialista na identificação de equipamentos e alarmes envolvidos (sintomas), associando-os com as prováveis explicações (causas).

Os sistemas especialistas geralmente apresentam uma arquitetura constituída por três módulos principais: uma base de conhecimento, um motor de inferência e uma interface com o usuário.

O conhecimento sobre o domínio é representado na base de conhecimento do SE, que é formada pela base de regras e pelos fatos, armazenados na memória de trabalho. Por outro lado, o motor de inferência é o mecanismo de controle do sistema que avalia e aplica as regras conforme as informações contidas na memória de trabalho. A arquitetura típica de um SE pode ser vista na Figura 1 (Bittencourt, 1998).

\subsubsection{Vantagens no uso de sistemas especialistas}

Entre as principais vantagens em se implementar um sistema especialista destaca-se:

- A redução de sobrecarga do operador, por excesso de alarmes, informações e restrições;

- Prevenção de erros humanos;

- Retenção do conhecimento da operação;

- Mais segurança e agilidade nas decisões operativas; 


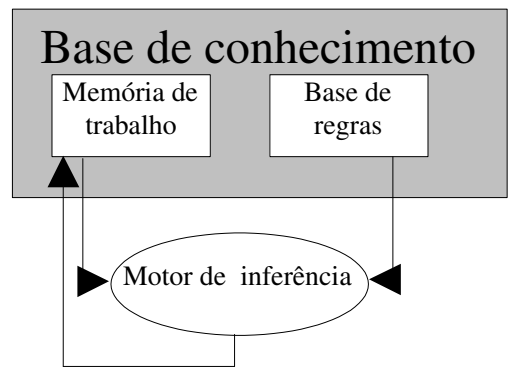

Figura 1: Arquitetura de um SE.

- Maior facilidade de aceitação da ferramenta pelos usuários, já que a mesma pode explicar a forma por meio da qual chegou a sua conclusão e funciona de forma semelhante ao raciocínio humano.

\subsubsection{Limitações dos sistemas especialistas}

Apesar dos sistemas especialistas constituírem uma solução válida, alguns fatores limitam a sua eficiência, sendo o principal a dificuldade, em algumas aplicações, de se adquirir, representar e validar o conhecimento de forma simples e completa.

- Na etapa de aquisição de conhecimento há necessidade de disponibilidade de um especialista humano com interesse em colaborar e que tenha facilidade de comunicação com o engenheiro de conhecimento, que desenvolverá o sistema especialista;

- Os sistemas especialistas não são capazes de generalizar, ou seja, limitam-se a responder de acordo com as informações contidas em sua base de conhecimento, sendo incapazes de tirar conclusões sobre algo que não esteja contido na mesma (Madan \& Bollinger, 1997);

- As ocorrências de faltas graves são raras, uma vez que os sistemas de energia são bastante confiáveis. Logo, nem mesmo um engenheiro experiente detém conhecimento sobre todos os tipos de faltas possíveis, sendo portanto difícil validar a base de conhecimento;

- Sistemas especialistas não devem conter informações completas (esgotamento de todas as possibilidades). Portanto, os projetistas são incapazes de prever sua eficiência em situações não testadas;

- Não apresentam bom desempenho quando a força bruta de busca não puder ser substituída por entendimento.

\subsubsection{Aplicação ao diagnóstico de faltas}

No caso do diagnóstico de faltas, os sistemas especialistas representam uma alternativa válida, porém têm problemas relativos a mudanças na topologia da rede, o que implica na formulação de um grande número de regras, consumindo muito tempo durante a fase de implementação. Outros problemas com relação a esta técnica são a dificuldade de lidar com dados corrompidos, e a necessidade de grande disponibilidade de um especialista humano.

Considere por exemplo, a proteção diferencial de barras, que tem por finalidade desligar todos os disjuntores conectados à barra. Neste caso, uma regra que corresponda a esta situação pode ser escrita como:

SE a proteção principal da barra (87) operou, E todos os disjuntores conectados à barra abriram, ENTÃO ocorreu uma falta na barra.

Considerando a possibilidade de falha de um ou mais disjuntores, a regra poderia ser escrita como:

SE a proteção principal da barra (87) operou, E nem todos os disjuntores conectados à barra abriram, $\mathbf{E}$ a proteção de falha de disjuntor operou, $\mathbf{E}$ operou a proteção de retaguarda remota (linha ou transformador adjacente à barra), ENTÃO ocorreu uma falta na barra.

Logo, as regras podem ser elaboradas com base na simulação da coordenação dos relés de proteção, considerando suas zonas de atuação e tempos de operação. Valores analógicos de corrente e tensão, também podem ser utilizados.

\subsection{Redes Neurais Artificiais}

As redes neurais artificiais (RNA) constituem uma forma de aproximação universal de funções, que apresenta um desempenho satisfatório durante a interpretação e classificação de padrões complexos. Estas são também bastante versáteis e podem aprender continuamente, além de apresentarem capacidade de generalização e alto desempenho computacional, devido ao seu forte paralelismo (Butler et al., 1997).

As redes neurais são inspiradas nos processos e estruturas cerebrais, sendo compostas por nós que recebem informações e as transferem mediante conexões ponderadas entre camadas, em geral definidas como de entrada, ocultas e de saída.

Geralmente, o neurônio (i) (Fig. 2) de uma determinada camada $(k)$ recebe informações de entrada, multiplica- 


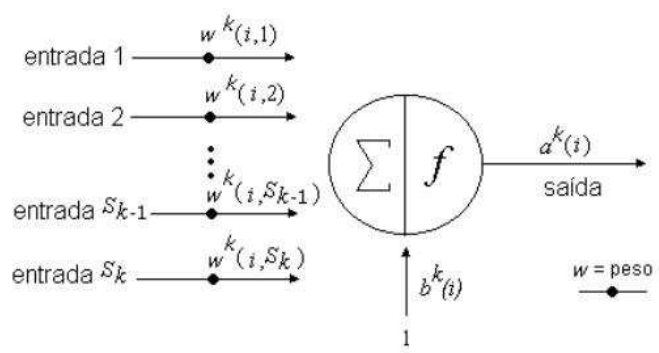

Figura 2: Nó de uma rede neural

as por cada um dos pesos $(w)$ correspondentes às conexões que receberam os dados, efetua o somatório $(\Sigma)$ destes produtos e processa esta somatória através de uma função de transferência $(f)$, produzindo um resultado $\left(a^{k}(i)\right)$. Geralmente é utilizado um valor auxiliar $b$ (bias) para representar uma polarização, valor abaixo do qual a saída é nula.

Uma rede neural é especificada com base nas características do problema a ser tratado, sendo esta treinada a partir de uma base de casos passados ou preparados como exemplos, de modo que a mesma seja capaz de estimar situações futuras.

\subsubsection{Vantagens}

- Capacidade de interpolação - as redes são capazes de produzir resultados mesmo com dados de entrada ruidosos e casos para os quais não foram treinadas, ou seja, insensibilidade ao ruído;

- Não necessitam de regras explícitas para a descrição do esquema de proteção. Somente dados sobre a operação de relés e disjuntores associados a uma determinada falta são necessários (Yang et al., 1994). Isto, pois o processo de aprendizagem da maioria das redes se dá através de exemplos;

- Podem aprender automaticamente novos cenários de falta, bastando adicionar-se novos dados ao conjunto de treinamento;

- Rapidez - o seu paralelismo intrínseco permite aumentar a velocidade de resposta;

- Fácil implementação - uma vez que a mesma utiliza exemplos durante o treinamento.

\subsubsection{Desvantagens}

- Padrões de treinamento dependem dos estados de relés e disjuntores, o que pode implicar em um aumento no número de conexões da rede neural, dificultando a sua aplicação em sistemas reais de grande porte. Kim \& Park (1993) sugerem que a estrutura da rede coincida com as características do sistema de potência, criando-se modelos específicos para linhas, transformadores e barras. Com isto, as dimensões da rede seriam menores e após ser treinada, a mesma não necessitaria ser atualizada, caso ocorram mudanças na configuração do sistema;

- Informações de estados de relés e disjuntores - Sun et al. (1998) consideram estas informações como sendo insuficientes, pois caso ocorra uma falta na linha de transmissão, os disjuntores e relés associados a esta linha podem falhar, operar incorretamente ou apresentar um desempenho anormal, prejudicando o diagnóstico final. Portanto, os mesmos sugerem que o sistema seja considerado como um todo, ou seja: linha de transmissão, disjuntores, relés, dispositivos de religamento e a seqüência de tempo em que cada evento ocorre;

- Dificuldade em se garantir um resultado confiável - pois é difícil especificar um conjunto de amostras (padrões de treinamento) significativas (Wen \& Chang, 1997);

- Sistemas de potência de grande porte requerem uma rede neural de grande escala, sendo difícil determinar os pesos ótimos em termos de precisão e esforço computacional, além de resultar em uma lenta convergência da rede durante o processo de treinamento. Portanto, a obtenção de modelos mais aceitáveis força uma redução na dimensão da rede (Mori, 1996);

- Necessidade de se determinar o tamanho da rede, ou seja, o número de neurônios e camadas da rede;

- Dados históricos contendo informações sobre faltas seriam o ideal para se treinar a rede, mas isto em uma aplicação real nem sempre está disponível, uma vez que as faltas ocorrem de maneira aleatória e não são tão freqüentes;

- Falta de critérios que determinem a quantidade de dados necessários ao aprendizado da rede. Poucos dados implicam em modelos inadequados, enquanto que muitos dados resultam em um treinamento bastante demorado e, por vezes, perda da capacidade de generalização (fenômeno conhecido como sobretreinamento);

- O algoritmo de retropropagação não é muito aceito, pois requer muitas iterações, sendo importante a utilização de algoritmo de aprendizado com boas características de convergência (Mori, 1996); 
- As redes não conseguem resolver eficientemente problemas em que a informação proveniente da seqüência de eventos compõe parte essencial para o diagnóstico.

\subsubsection{Aplicação ao diagnóstico de faltas}

No caso do diagnóstico de faltas, o problema é formulado como um reconhecimento de padrões, onde diferentes combinações de estados de relés e disjuntores são identificadas (Yang et al., 1994).

Na primeira camada tem-se um nó para cada variável de entrada (Fig. 3). A informação é recebida por estas entradas, que por sua vez a encaminham aos neurônios, que constituem a camada oculta, que assim como os neurônios da camada de saída recebem a informação, realizam processos de multiplicação e acumulação, e por meio de uma função de transferência produzem resultados. Estes são comparados com os valores esperados, encontrando-se as diferenças que constituem um vetor de erros. As derivadas deste vetor em função dos respectivos pesos são determinadas, sendo transferidas, no sentido inverso, voltando aos neurônios da camada oculta (processo de retropropagação). Nestes neurônios calcula-se a somatória das derivadas dos erros, definindo-se sua participação no erro de saída. Mediante algum procedimento matemático, como o método dos mínimos quadráticos, os neurônios modificam os pesos de suas conexões, diminuindo o erro apresentado pela rede. O processo repete-se ciclicamente, até que os neurônios da camada de saída apresentem um erro inferior ao estipulado (Lefèvre, 1996).

As redes neurais diferem bastante das técnicas de IA que dependem do conhecimento prévio das regras que governam o fenômeno, pois são adaptáveis e inferem soluções a partir dos exemplos utilizados durante o treinamento. $\mathrm{O}$ fato de estas serem não lineares permite que elas captem interações complexas entre as variáveis, como ocorre normalmente no mundo real

Entre os principais problemas a serem levados em consideração durante a implementação de redes neurais em diagnóstico de faltas estão:

- Sistemas de potência de grande porte podem requer uma rede neural de grande escala, sendo difícil determinar os pesos ótimos em termos de precisão e esforço computacional (Mori, 1996).

- Sistemas elétricos de potência são tipicamente grandes, existem inúmeros possíveis locais de faltas e, a cada uma delas relacionam-se determinados dispo-

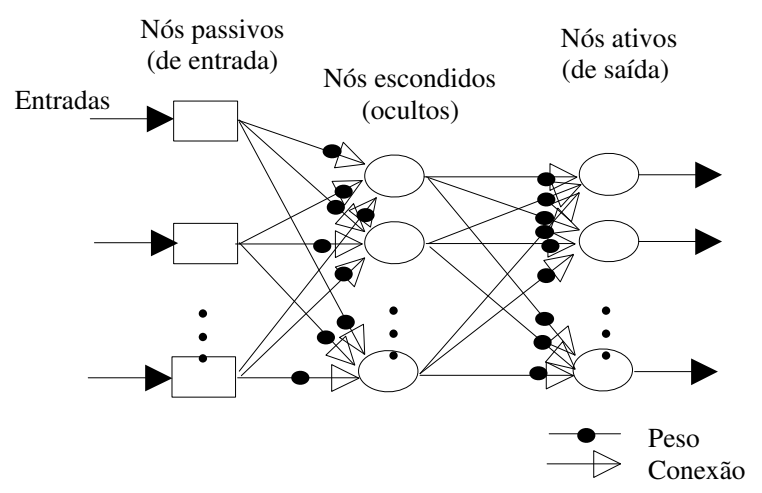

Figura 3: Rede neural elementar.

sitivos de proteção. Tornam-se impraticáveis os padrões representativos a serem utilizados durante o treinamento das mesmas, principalmente no caso de faltas múltiplas. Isto resulta em uma lenta convergência da rede durante o processo de treinamento, sendo, portanto essencial reduzir ao máximo o número de neurônios da camada de entrada (Rodriguez et al., 1996).

- Obtenção de modelos mais aceitáveis força uma redução na dimensão da rede.

- Os sistemas elétricos de potência freqüentemente sofrem mudanças de topologia, seja por razões de manutenção ou questões de eficiência. Portanto, o uso de uma simples grande rede neural, na qual a flexibilidade é reduzida, não é útil a um sistema de potência real (Rodríguez et al., 1996). Para Coutto et al. (1999) uma única rede para todo o sistema considera uma topologia fixa, e necessita ser treinada após cada mudança topológica, tornando-se inviável (Mori, 1996).

- Dados históricos contendo informações sobre faltas seriam o ideal para se treinar a rede, mas isto em uma aplicação real nem sempre está disponível, pois as faltas ocorrem de maneira aleatória e não são tão freqüentes (Rodríguez et al., 1996). É importante, durante a fase de treinamento da rede, evitar que a mesma seja suprida de informações conflitantes.

- Falta de critérios que determinem a quantidade de dados necessários ao aprendizado da rede. Poucos dados implicam em modelos inadequados, enquanto que muitos dados resultam em muito tempo computacional durante o treinamento.

- O algoritmo de retropropagação não é muito aceito, pois requer muitas iterações, sendo importante a 
utilização de algoritmo de aprendizado com boas características de convergência (Mori, 1996).

As redes neurais são bastante promissoras, pois possuem excelente capacidade de generalização; podem ser implementadas com maior facilidade, uma vez que aprendem por exemplos, e apresentam grande rapidez na solução do problema. Porém, é necessário modelar melhor o problema, de modo a diminuir ao máximo o número de neurônios na camada de entrada, e evitar que a eficiência da rede seja prejudicada por possíveis mudanças na topologia do sistema monitorado. Outra dificuldade a ser levada em consideração é a questão do raciocínio temporal.

\subsection{Algoritmos genéticos}

Os Algoritmos Genéticos (AGs) são algoritmos de otimização e busca baseados nos mecanismos de seleção natural e genética. A teoria emula o processo da natureza onde os mais aptos vencem e se reproduzem e, conseqüentemente, os mais fracos se extinguem. O método consiste inicialmente de uma população formada por uma série de bits (string - representando os cromossomos), que é transformada por três operadores genéticos: seleção, reprodução e mutação (Goldberg, 1989).

Cada string (cromossomo) representa uma possível solução do problema a ser otimizado, e cada bit (ou grupo de bits), representa o valor associado a determinadas variáveis do problema (gene). As soluções são classificadas por uma função de aptidão (fitness) que desempenha o papel do ambiente. O par cromossomo e função de aptidão representam o indivíduo (Goldberg, 1989).

Recentemente, esta nova classe de métodos baseados em técnicas de otimização tem sido aplicada ao problema de diagnóstico de faltas. Embora resultados de simulações mostrem que este método é bastante promissor em sistemas de potência de grande porte, nem sempre é fácil estabelecer um modelo matemático capaz de descrever o comportamento do sistema de proteção (Wen \& Chang, 1997).

O interesse por este algoritmo tem aumentado de forma significativa, uma vez que o mesmo fornece um mecanismo de busca bastante robusto, além de se basear em importantes conceitos biológicos (Miranda et al., 1996).

Os algoritmos genéticos como sendo um processo onde se gera uma população inicial aleatoriamente, avalia-se esta, e criam-se novas populações por meio de operadores genéticos. Os indivíduos com bom desempenho são selecionados e partes destes são combinadas, criando cópias mais fortes, simplesmente pelo uso de seleção e reprodução. Apesar desta aparente simplicidade, cabe ressaltar que o processo de busca exige uma substancial força computacional que podem inviabilizar sua utilização em aplicações on-line (Wen \& Han, 1995).

Os modelos apresentados na literatura são bastante promissores, pois independem da topologia da rede. Porém, alguns destes utilizam dados sobre a confiabilidade de operação dos dispositivos de proteção, que nem sempre estão disponíveis e são de difícil aquisição (dependem do modelo e fabricante, da manutenção, tempo de uso, atualização da coordenação dos dispositivos de proteção).

Dependendo do grau de depreciação dos alarmes recebidos, as formulações até então estudadas apresentam dificuldades em produzir um diagnóstico correto.

\subsection{Redes de Petri}

As redes de Petri são redes com ponderações nas relações de fluxo, onde o elemento $s$ é chamado de lugar (pode ser interpretado como uma condição, um estado parcial, uma espera, um procedimento, entre outros), ao invés de estado. É bastante comum representar a rede de Petri por grafos constituídos de nós lugares e nós transições. Tais nós são interligados por meio de um ou mais arcos (Cardoso \& Valette, 1997).

Mais precisamente, as redes de Petri podem ser consideradas como sendo uma ferramenta gráfica com grande capacidade para modelar e analisar sistemas a eventos discretos. Adicionalmente, as redes de Petri podem ser utilizadas para simular as atividades dinâmicas e concorrentes dos sistemas (Wang \& Tang, 1997).

Segundo Lo et al. (1997), as redes de Petri constituem uma excelente ferramenta para modelar o sistema de proteção. O processo de diagnóstico consiste em manipulações matriciais bastante simples e apresenta bons resultados.

\subsubsection{Uma breve revisão sobre as redes de Petri}

As redes de Petri (Figura 4) são constituídas por dois tipos de nós e arcos. Os nós correspondem a dois conjuntos disjuntos: lugares $S$, tal que, $S=\left\{s 1, s 2, \ldots, s_{m}\right\}, m$ $>0$, representados por círculos "O"; transições $T$, considerando que $T=\left\{t 1, t 2, \ldots, t_{n}\right\}, n>0$, representados por barras "|". Os arcos indicam a direção do processo e correspondem a dois conjuntos disjuntos: arcos de entrada e de saída. Os arcos de entrada são indicados por um arco direcionado de $\mathrm{s}_{i}$ (lugar) a $t_{j}$ (transição), enquanto que os de saída são indicados por um arco direcionado 
da transição para o lugar (Wang \& Tang, 1997).

Utilizando-se os conceitos de estados e eventos, os lugares representam os estados, e as transições, os eventos. Os lugares de entrada e saída da transição representam os estados antes e após a ocorrência do evento, respectivamente.

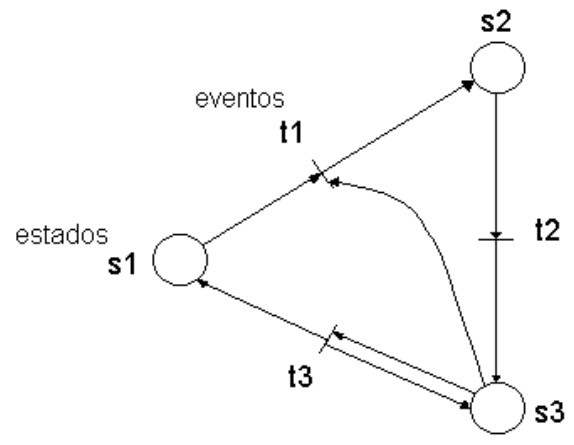

Figura 4: Grafo de uma rede de Petri (Lo et al., 1997).

A topologia de qualquer rede de Petri pode ser representada pela matriz de incidência (equação 1) $C$, com dimensão ( ${ }^{o}$ de lugares) x ( ${ }^{o}$ de transições $)$, ou seja ,

$$
C(s, t)= \begin{cases}-W(s, t) & (s, t) \in F \\ +W(s, t) & (t, s) \in F \\ 0 & \text { seno }\end{cases}
$$

onde,

$W(s, t)$ - peso do arco que liga $s$ a $t$.

$(s, t) \in F$ - significa que existe uma relação de fluxo $(F)$ de $s$ para $t$.

$(t, s) \in F$ - significa que existe uma relação de fluxo $(F)$ de $t$ para $s$.

A rede de Petri marcada é uma rede de Petri com a introdução de fichas (indicador de que a condição associada ao lugar é verificada), K(s), em um lugar e com uma marcação inicial, $\mathrm{M}_{0}(\mathrm{~s})$. $\mathrm{K}(\mathrm{s})$ pode ser qualquer número natural, e representa o número máximo de fichas que um lugar (s) pode possuir.

As redes de Petri marcadas são utilizadas para modelar o sistema de proteção. Neste caso, os elementos de $S$, correspondem às linhas de transmissão, barras, relés e disjuntores. Por outro lado, os elementos de $T$ representam a transição da falta, e o período de tempo entre a detecção da falta, por parte do relé e a efetiva operação dos disjuntores.

\subsubsection{Regras de execução}

A propriedade dinâmica da rede é definida pelas marcas e execução da rede, sendo a estrutura da rede estática. A execução corresponde ao disparo de uma transição que muda as fichas de um lugar para outro.

A marca final resultante do disparo de uma série de transições pode ser expressa pela equação 2 .

$$
M_{1}(s)=M_{0}(s)+\left[-W\left(s, t_{k}\right)+W\left(t_{k}, s\right)\right], \quad \forall s \in S
$$

Considerando que:

$M_{0}(s)$ - marca inicial (depende somente dos disjuntores);

$M_{1}(s)$ - marca final;

$t_{k}$ - disparo da k-ésima transição;

$S$ - conjunto contendo todos os lugares.

Definindo um vetor $U,\left[U_{1} U_{2} \ldots U_{n}\right]^{T}$, onde, $U_{n}$ é o número de ocorrências de $t_{n}$, a equação 2 pode ser escrita como o apresentado pela equação 3 .

$$
M_{1}=M_{0}+C * U
$$

O processo de diagnóstico de faltas ocorre no sentido inverso daquele referente à eliminação da falta. Neste caso, é utilizado o modelo de rede de Petri reversa, onde o estado inicial da rede corresponde ao estado final da proteção, ou seja, a marcação inicial depende somente de informações sobre os estados dos disjuntores.

As redes de Petri são simuladas somente para as partes do sistema de potência atingidas pela falta (aquelas em que houve desligamento).

\subsubsection{Problemas a serem solucionados}

Em linhas gerais, embora as redes de Petri mostrem-se bastante promissoras, existem alguns aspectos relativos aos modelos propostos em artigos que necessitam ser melhorados, ou seja:

- Caso os relés e disjuntores não estejam em boas condições de operação, os modelos apresentam dificuldades em produzir diagnóstico correto;

- A representação gráfica do esquema de proteção, que aparentemente implica em grande facilidade em se visualizar a operação da proteção, perde a importância quando se trata de sistemas de potência reais (grande dimensão); 
- O mapeamento de sistemas complexos por meio de redes de Petri é bastante difícil, sendo ainda motivo de pesquisa.

Os modelos encontrados na literatura parecem ser bastante simples, mas a resposta final nem sempre representa a solução do problema, a qual ainda necessita de uma melhor interpretação. Entre os modelos estudados, nenhum corresponde ao esquema de proteção completo (por ex., no caso das linhas, não consideram o esquema de teleproteção, proteção de retaguarda local e remota, etc.), sendo estes impraticáveis em situações reais.

\subsection{Lógica Difusa}

A lógica difusa consiste em aproximar a decisão computacional à decisão humana. Isto é feito de forma que a decisão de uma máquina não se resuma apenas a um "sim" ou um "não", mas também tenha decisões "abstratas", do tipo "um pouco mais", "talvez sim", e outras variáveis que representem as decisões humanas. A lógica difusa tem se destacado principalmente na área de tomada de decisões e controle (Tafner et al., 1996).

A lógica difusa constitui um excelente suporte na elaboração de modelos completos e eficientes, capazes de representar a incerteza e a imprecisão do raciocínio humano, onde variáveis lingüísticas associadas a funções de pertinência são utilizadas. O processo de fuzzification oferece uma maior robustez e capacidade de generalização, facilitando a modelagem de problemas complexos, com baixo custo de solução. Tais razões propiciaram um aumento na preferência de sistemas baseados em lógica difusa na solução de problemas em sistemas de potência (Srinivasan et al., 1995).

Do ponto de vista do diagnóstico de faltas, somente as partes das regras relativas às conclusões são conhecidas em forma de alarmes, sendo basicamente impossível identificar a seção em falta por meio de raciocínio dedutivo. Portanto, o problema de diagnóstico de faltas é um processo de raciocínio indutivo por natureza, onde deve ser considerada a possibilidade de mau funcionamento dos dispositivos de proteção. As incertezas no diagnóstico de faltas surgem uma vez que as premissas (causas) das regras devem ser inferidas utilizando informações das partes relativas às conclusões (efeitos) em situações em que pode ocorrer o mau funcionamento de componentes (Park et al., 1999).

Parte das regras utilizadas durante o diagnóstico não são crisp, isto é, geralmente as decisões são baseadas no senso comum, sendo perfeitamente possível a utilização de variáveis difusas (Monsef et al., 1997).

\subsubsection{Vantagens}

Para Chang et al. (1997), entre as principais vantagens apresentadas pelos sistemas baseados em lógica difusa, destacam-se:

- Flexibilidade - as funções de pertinência utilizadas para representar a performance de relés e disjuntores podem ser alteradas dinamicamente de acordo com as condições de falta, condições do tempo e disponibilidade de dados estatísticos;

- Capacidade de supressão de ruídos - não é sensível a sinais inesperados, tais como falha ou operação indevida de relés, erros na transmissão de dados, entre outros;

- Geralmente, o processo de inferência utiliza um pequeno número de regras nebulosas e a ordem de disparo das mesmas não influi no resultado, o que torna fácil adicionar novas regras na base de conhecimento.

\subsubsection{Características que combinam com lógica di- fusa}

Desde 1984 vem crescendo significativamente o uso de lógica nebulosa em sistemas de potência, seja nas áreas de controle, planejamento e operação. Este súbito interesse surge devido ao pouco esforço computacional envolvido e à simplicidade de implementação. Outro aspecto atrativo é o fato destes sistemas serem bastante robustos em aplicações envolvendo informações imprecisas e vagas, além de aplicações envolvendo objetivos conflitantes (Srinivasan et al., 1995). Algumas características e requisitos comuns à escolha da lógica nebulosa são:

- Disponibilidade de regras heurísticas (necessidade da ajuda de especialistas humanos);

- Inexistência de expressão em forma matemática como método de solução;

- A modelagem matemática do problema requer várias simplificações, resultando em um modelo pouco preciso;

- O problema envolve restrições vagas e/ou múltiplos objetivos conflitantes; 
- A complexidade do problema torna a solução computacional bastante pesada, caso técnicas convencionais sejam utilizadas.

A lógica difusa parece ser uma alternativa bastante promissora, pois apresenta grande habilidade em tratar informações imprecisas, captar informações de senso comum, tomar decisões, além da capacidade em lidar com raciocínios envolvendo linguagem natural.

\section{CONCLUSÕES}

Este trabalho procurou introduzir o problema de diagnóstico de faltas, definindo e ressaltando algumas características peculiares apresentadas pelo mesmo. Tal abordagem teórica foi idealizada com o objetivo de fornecer conhecimentos sobre as alternativas para tratamento do problema a quem porventura venha atuar nesta área.

É interessante que as explicações finais da ferramenta de diagnóstico não apenas indiquem os elementos (barra, linhas, transformadores, etc.) em falta, mas também, os relés e disjuntores que falharam ou operaram indevidamente, de modo que estes possam ser encaminhados à equipe de manutenção. Assim, pode-se melhorar a eficiência da proteção do sistema de potência, quando da ocorrência de futuras faltas.

As diversas técnicas encontradas na literatura diferem bastante uma da outra, porém, a principal etapa do diagnóstico é comum a todos, ou seja, oconhecimento sobre a operação do sistema elétrico a ser monitorado. Sendo que no caso dos sistemas especialistas, o conhecimento está representado em forma de regras; nas redes neurais, no conjunto de exemplos utilizados durante a fase de treinamento; nos algoritmos genéticos e busca tabu, na função de aptidão ou objetivo; nas redes de Petri, na própria representação da rede; nos conjuntos difusos, na função de pertinência e nas regras a serem disparadas.

Por fim, a capacidade ou eficiência de cada um destes sistemas inteligentes irá depender do tipo de dados disponíveis, e de como o problema é formulado ou modelado.

\section{REFERÊNCIAS}

Bell, S.C.; McArthur, S. D. J.; McDonald, J. R. et al.; 1998. Model-based analysis of protection system performance. IEE Proc.-Gener. Transm. Distrib., Vol. 145, No. 5 (Sept.), p. 547-552.

Bittencourt, G; 1998. Inteligência Artificial: ferramen- tas e teorias. $1^{a}$ Edição. Florianópolis: Ed. da UFSC.

Butler, K. L.; Momoh, J. A.; Sobajic, D. J.; 1997. Field studies using a neural-net-based approach for fault diagnosis in distribution networks. IEE Proc.Gener. Transm. Distrib., Vol. 144, No. 5 (Sept.), p. 429-436.

Cardoso, J. \& Valette, R.; 1997. Redes de Petri. $1^{a}$ Edição. Florianópolis: Editora da UFSC. p. 220.

Chairman, J. A. B.; Chairman, R. O. B. V.; Baugartner, E. A. et al.; 1998. Fault and Disturbance Data Requirements for Automated Computer Analysis Summary Paper. IEEE Transaction on Power Delivery, Vol. 13, No. 3 (July), p. 735-737.

Chang, C. S. \& Wen, F. S.; 1998. Tabu search based approach to trouble call analysis. IEE Proc.-Gener. Transm. Distrib., Vol. 145, No. 6 (Nov.), p. 731-738.

Chang, C. S.; Chen, J. M.; Srinivasan, D. et al.; 1997. Fuzzy logic approach in power system fault section identification. IEE Proc.-Gener. Transm. Distrib., Vol. 144, No. 5 (Sept.), p. 406-414.

Chiganer, L.; Biondi Neto, L.; Fukuda, F. H. et al.; 2000. Electrical Networks Fault Diagnosis a Neural Approach. In: VII Symposium of Specialists in Electrical Operational and Expansion Planning (May. 2000: Curitiba, Paraná). p.1-6.

Coutto Filho, M. B. do; Rodrigues, M. A. P.; Souza, J. C. S. et al.; 1999. Localização de defeitos em sistemas de energia elétrica utilizando sistemas inteligentes. In: XV SNPTEE, Seminário Nacional de Produção e Transmissão de Energia Elétrica (Outubro 1999: Paraná, Brasil). p.1-7.

Dyliacco, T. E. \& Kraynak, T. J.; 1969. Processing by logic programming of circuit breaker and protective relaying information. IEEE Trans. On Power Apparatus and Systems, Vol. PAS-88, No. 2 (Feb.).

Feigenbaum, E. A., 1982. Knowledge engineering in the 1980s. Dept. of Computer Science, Stanford University, Stanford, CA.

Fu, S.; Wang, P.; Wang, M. et al.; 1995. An Expert System for on-line Diagnosis of System Faults and Emergency Control to Prevent a Blackout. In: IFAC Control of Power Plants and Power Systems, SIPOWER'95 (Cancun, Mexico). p. 303-308.

Goldberg, D. E; 1989. Genetic algorithms in search, optimization, and learning. Addison-Wesley, Reading, MA. 
Gomes, N. S.; Andrade, S. R. C.; Cota, C. A. B. et al.; 1999. SAPNET - Sistema de Análise de Perturbações da CEMIG. In: XV SNPTEE, Seminário Nacional de Produção e Transmissão de Energia Elétrica (Outubro 1999: Paraná, Brasil). p. 1-5.

Gomes, P.; Marangon Lima, J. W.; Schilling, M. Th.; 2002. Estratégias para Aumento da Segurança da Malha Elétrica Nacional: Lições Extraídas dos Grandes Blecautes. In: VIII SEPOPE, Simpósio de Especialistas em Planejamento da Operação e Expansão Elétrica (Maio 2002: Brasília, Brasil).

Handschin, E.; Kuhlmann, D.; Hoffmann, W.; 1996. System Fault Diagnosis. University of Dortmund. Edited by M. A. El-Sharkawi and Dagmar Niebur, Chapter 11. p. 138-149.

Hertz, A. \& Fauquembergue, P.; 1992. Fault Diagnosis at Substations Based on Sequential Event Recorders. Proceedings of the IEEE, Vol. 80, No. 5 (May), p. $684-688$.

Jota, F. G. \& Jota, P. R. S.; 1998. High-impedance fault identification using a fuzzy reasoning system. IEE Proc.-Gener. Transm. Distrib., Vol. 145, No. 6 (Nov.), p. 656-662

Kim, K. H. \& Park, J. K.; 1993. Application of hierarchical neural networks to fault diagnosis of power systems. Electric Power \& Energy Systems, Vol.15, No. 2, p. 65-70.

Lefévre, M. A. P.; 1996. Sistemas Inteligentes e sua Aplicação na Operação de Itaipu Binacional. Eletroevolução, No. 7 (Out.), P. 54-58.

Lo, K. L.; Ng, H. S.; Trecat, J.; 1997. Power system fault diagnosis using Petri nets. IEE Proc.-Gener. Transm. Distrib., Vol. 144, No. 3 (May), p. 231-236.

Madan, S. \& Bollinger, K. E.; 1997. Application of artificial intelligence in power systems. Electric Power Systems Research, 41, p. 117-131.

Miranda, V.; Srinivasan, D.; Proença, L. M.; 1996. Evolutionary computation in power systems. $12^{\text {th }}$ Power Systems Computations Conference, Dresden (Aug, 19-23), p. 25-40.

Mohamed, E. A. \& Rao, N.D.; 1995. Artificial neural network based fault diagnosis system for electric power distribution feeders. Electric Power Research, 35, p. 1-10.

Monsef, H.; Ranjbar, A. M.; Jadid, S.; 1997. Fuzzy rulebased expert system fault diagnosis. IEE Proc.Gener. Transm. Distrib., Vol. 144, No. 2 (Mar.), p. 186-192.
Montakhab, M. R. \& Adams, R. N.; 1998. Intelligent system for fault diagnosis on low voltage distribution networks. IEE Proc.-Gener. Transm. Distrib., Vol. 145, No. 5 (Sept.), p. 592-596.

Mori, H.; 1996. State-of-the-art Overview on Artificial Neural Networks in Power Systems. Meiji University, Edited by M. A. El-Sharkawi and Dagmar Niebur, Chapter 6. p. 51-58.

Murta Vale, M. H.; Parma, G. G. et al.; 2002. STA Alarm Processing System Computational Tool Integrated into CEMIG Supervisory Control System. In: VIII SEPOPE, Simpósio de Especialistas em Planejamento da Operação e Expansão Elétrica (Maio 2002: Brasília, Brasil).

Park, D. Y.; Ahn, B. S.; Kim, S. H. et al.; 1999. Dealing Uncertainties in the Fault Diagnosis System. In: Intelligent System Application to Power Systems (ISAP'99 April 4-8, 1999: Rio de Janeiro, Brazil). p. 273-277.

Rodrigo, P. D.; Pahwa, A.; Boyer, J. E.; 1996. Location of outages in distribution systems based on statistical hypotheses testing. IEEE Transactions on Power Delivery, Vol. 11, No. 1 (Jan.), p. 546551.

Rodriguez, C.; Rementeria, S.; Martin, J. I. et al.; 1996. Fault analysis with modular neural networks. Electric Power 83 Energy Systems, Vol.18, No. 2, p. 99110.

Srinivasan, D.; Liew A. C.; Chang, C. S.; 1995. Applications of fuzzy systems in power systems. Electric Power Systems Research, 35, p. 39-43.

Sun, Y.; Jiang, H.; Wang, D.; 1998. Fault synthetic recognition for an EHV transmission line using a group of neural networks with a time-space property. IEE Proc.-Gener. Transm. Distrib., Vol. 145, No. 3 (May), p. 265-270.

Tafner, M. A.; Xerez, M. de.; Rodrigues Filho, I. W.; 1996. Redes neurais artificiais: introdução e princípios de neurocomputação. Ed. da FURB, p. 199.

Teo, C. Y. \& Gooi, H. B.; 1998. Artificial intelligence in diagnosis and supply restoration for a distribution network. IEE Proc.-Gener. Transm. Distrib., Vol. 145 , No. 4 (July), p. 444-450.

Terreros, A. J. P.; Sell, H. L.; Eidt, J. F.; 1999. Implantação de Oscilografia Digital na ELETROSUL. In: XV SNPTEE, Seminário Nacional de Produção e Transmissão de Energia Elétrica (Outubro de 1999: Paraná, Brasil). p. 1-6. 
Vale, Z. A. \& Ramos, C.; 1995. Temporal Reasoning in AI Applications for Power System Control Centers. In: IFAC Control of Power Plants and Power Systems (SIPOWER'95), Cancun, Mexico). p. 297302.

Wang, F. \& Tang, J.; 1997. Modeling of a transmission line protective relaying scheme using Petri nets. IEEE Trans. Power delivery, Vol. 12, No. 3, p. 1055-1063.

Wen, F. \& Han, Z.; 1995. Fault section estimation in power systems using a genetic algorithm. Electric Power Systems Research, 34, p. 165-172.

Wen, F. S. \& Chang, C. S.; 1997. Probabilistic approach for fault-section estimation in power systems based on a refined genetic algorithm. IEE Proc.-Gener. Transm. Distrib., Vol. 144, No. 2 (Mar.), p. 160168.

Wollenberg, B.; 1986 Feasibility study for an energy management system intelligent alarm processor. IEEE Trans. Power Syst., Vol. 1 (May), p. 241-247.

Yang, H. T. ;Chang, W. Y.; Huang, C. L.; 1994. A new neural network approach to on-line fault section estimation using information of protective relays and circuit breakers. IEEE Transaction on Power Delivery, Vol. 9, No. 1 (Jan.), p. 220-229. 